\title{
Developmental Changes in the Relative Weighting of Geometric and Experience-Dependent Location Cues
}

\author{
Alycia M. Hund and John P. Spencer \\ Department of Psychology \\ University of Iowa
}

\begin{abstract}
According to the category adjustment model of location estimation, children's responses are biased toward spatial prototypes, and these biases increase under conditions of uncertainty. Consistent with the model, 6- and 11-year-olds' biases toward prototypes increased across delays, especially for locations far from prototypes. Response biases also varied systematically with target frequency; however, responses were not always biased toward prototypes. In Experiment 1, 6-year-olds' responses to an infrequent target near the category boundary were biased toward a frequent target in an adjacent category. In Experiment 2, biases toward a frequent target were evident near prototypes. Both categorical information and children's experience with locations influence location estimates. Moreover, children's selective weighting of competing location cues changes between 6 and 11 years.
\end{abstract}

Spatial cognition has played a central role in developmental psychology since Piaget's early explorations of children's understanding of space (Piaget \& Inhelder, 1956; Piaget, Inhelder, \& Szeminska, 1960). Despite this long history of research, the field lacks a unifying theory of the development of spatial skills. Recently, Newcombe, Huttenlocher, and colleagues (Newcombe \& Huttenlocher, 2000; Newcombe, Huttenlocher, Drummey, \& Wiley, 1998) proposed a framework that may unify decades of spatial memory research. According to their account, children encode locations using four coding systems: response learning, dead reckoning, cue learning, and place learning. The response-learning and dead-reckoning systems encode locations in relation to the self. In contrast, the cue-learning and place-learning systems encode locations relative to landmarks in

Requests for reprints should be sent to Alycia M. Hund, Department of Psychology, 11 Seashore Hall E., University of Iowa, Iowa City, Iowa 52242. E-mail: alycia-hund@uiowa.edu 
the environment. When estimating a location, children use a "weighting" process to combine location information obtained from these four coding systems. For instance, when searching for a toy hidden in a large sandbox, children rely primarily on "place" information, encoding the metric distance between a hidden toy and the edges of the sandbox (Huttenlocher, Newcombe, \& Sandberg, 1994; Newcombe et al., 1998; see also Spencer, Smith, \& Thelen, 2001). From this coding systems perspective, developmental changes in spatial memory abilities reflect changes in the relative weighting of different location cues. That is, children learn which cues or combinations of cues lead to accurate, efficient responses in a given context.

The place-learning system is perhaps the most powerful and flexible of the four systems described by Newcombe and Huttenlocher (2000). As such, it has been the focus of a great deal of developmental research (e.g., Newcombe et al., 1998; Overman, Pate, Moore, \& Peuster, 1996; Sandberg, Huttenlocher, \& Newcombe, 1996). Moreover, Huttenlocher, Hedges, and Duncan (1991) proposed a category adjustment (CA) model of location memory that captures the characteristics of this system. Although findings from numerous studies support the model's claims regarding the types of information children use to estimate locations and changes in how these cues are used over development (e.g., Huttenlocher et al., 1994; Sandberg et al., 1996), few studies to date have formally tested specific predictions derived from the model. The goal of this study was to test two predictions of the CA model regarding how certainty affects location memory. In particular, we investigated how children's memory for locations changes as a function of the length of the memory delay in spatial recall tasks, and the frequency with which children respond to each location. Our results demonstrate that the CA model can account for many aspects of children's recall performance. Furthermore, these data suggest that the weighting perspective proposed by Newcombe and Huttenlocher (2000) is particularly useful when location cues are put in conflict. Our results also show dramatic experience-dependent effects in children's recall performance.

\section{THE CATEGORY ADJUSTMENT MODEL}

According to the CA model, children and adults encode location information at two levels of detail. At the first level, people encode the fine-grained or metric location of a target. Fine-grained information is encoded along continuous spatial dimensions such as the direction and distance from a reference location. At the second level, people encode the region or category in which the target is located. Categorical information is specified by boundaries that divide space into regions and spatial prototypes located at the geometric centers of regions. When asked to reproduce a location, people combine fine-grained and categorical information. This leads to systematic biases in location memory: responses within a category 
are biased toward the category center (prototype) because all locations within a category are weighted with the same prototype.

Findings from numerous studies support the proposal that children and adults use fine-grained and categorical information to estimate locations (e.g., Engebretson \& Huttenlocher, 1996; Huttenlocher et al., 1991; Laeng, Peters, \& McCabe, 1998; Newcombe et al., 1998; Newcombe, Huttenlocher, Sandberg, Lie, \& Johnson, 1999; Plumert \& Hund, 2001; Sandberg et al., 1996). For instance, when asked to remember the angular location of a line inside a small, inverted $\mathrm{V}$ frame, 7- and 9-year-olds' responses were clustered around the actual angular location, suggesting that they used fine-grained information to estimate the location (Sandberg et al., 1996). However, responses were biased toward the centers of the left and right categories in the $V$ (see Figure 1, for similar results with adults; see Engebretson \& Huttenlocher, 1996). That is, children's responses were biased toward the centers of the geometric categories formed using the midline axis as a category boundary.

In addition to demonstrating that children and adults use fine-grained and categorical information, previous studies have revealed important developmental changes in the ways children categorize space (e.g., Huttenlocher et al., 1994; Sandberg, 1999; Sandberg et al., 1996). For example, Huttenlocher and colleagues (1994) found that children between the ages of 2 and 6 years use visible category boundaries - the edges of a 5-foot-long rectangular sandbox-to help them remember locations. As a result, their responses were biased toward the center of the sandbox (the center of the large category). By contrast, 10-year-olds' responses were biased toward the centers of the two halves of the sandbox, suggesting that they subdivided the box into two categories. These findings suggest that children's

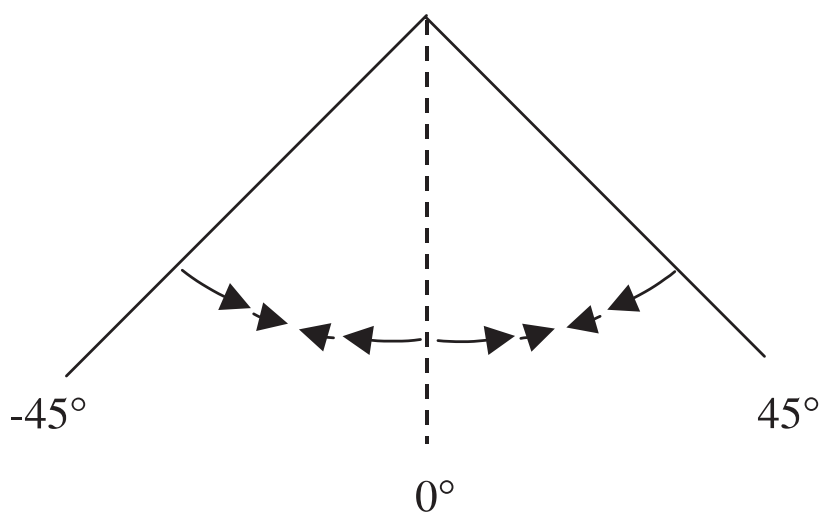

FIGURE 1. Schematic of biases for remembered locations in the V-frame task from Sandberg et al. (1996). The arrows indicate biases toward category prototypes. The dashed midline symmetry axis $\left(0^{\circ}\right)$ is shown for illustrative purposes only-it was not present in the task space. 
ability to subdivide space into multiple categories undergoes qualitative developmental changes between 6 and 10 years, although the precise age at which this change in skill occurs appears to depend on the size of the space to be subdivided (see Huttenlocher et al., 1994, Experiment 6).

As this review suggests, the majority of studies investigating the CA model to date have focused on how children and adults use geometric category information to remember locations. Across studies, children and adults consistently use the visible edges of a geometric figure (or the edges of the task space) and symmetry axes to divide space into regions. Moreover, recall responses are systematically biased away from these geometric category boundaries toward the center of each resulting region, that is, toward spatial prototypes. Although geometric category information clearly has a major impact on location estimation, other types of location cues may modulate recall responses. Children and adults might, for instance, construct an accurate or certain fine-grained representation of each target location as they respond repeatedly to the same targets. Children and adults might also group locations that are close together or that have been experienced close together in time (e.g., Hund, Plumert, \& Benney, 2002; McNamara, Halpin, \& Hardy, 1992). These examples raise important questions about people's ability to use task-specific experience to estimate locations. This study takes a first step toward exploring such experience-dependent effects by relating these possibilities directly to predictions of the CA model. In particular, we examine how experience might modulate the certainty of fine-grained information and the relative balance between experience-dependent groups of locations and spatial prototypes.

Although experience-dependent effects have not been directly examined in the context of the CA model, such effects are conceptually related to one factor that influences how children and adults combine location cues-the certainty of each cue (Engebretson \& Huttenlocher, 1996; Huttenlocher et al., 1991; Newcombe et al., 1999). As the certainty of fine-grained information about a location decreases, the weighting of categorical information increases, leading to greater bias toward spatial prototypes. Conversely, when fine-grained information is relatively certain, categorical information receives a low weight, resulting in minimal bias. In addition to affecting the magnitude of bias, certainty influences the variability of responses. Variability is relatively low when people are certain about locations. However, variability is higher when people are less certain about locations, especially when they are uncertain about a location's category membership (i.e., when locations are very near category boundaries).

Despite these specific proposals regarding the effects of memory certainty, few researchers have investigated how certainty influences recall responses. One exception is work by Engebretson and Huttenlocher (1996). These researchers explored whether adults' biases toward category prototypes depended on the certainty of fine-grained information. To manipulate fine-grained certainty, adults were asked to remember a distractor location between the presentation of a target 
and the recall response. As predicted by the CA model, biases toward spatial prototypes were larger in the interference condition relative to a noninterference condition, suggesting that when the fine-grained memory of a target location is degraded, adults rely more heavily on prototypical information (see also Hund \& Plumert, 2002; Huttenlocher et al., 1991). In this study, we examined how the certainty of location information affects children's ability to remember locations. In particular, we explored how the length of memory delays and the frequency with which children responded to locations influenced their ability to combine fine-grained and categorical information in a spatial recall task.

\section{SPECIFIC GOALS}

This investigation tested two predictions of the CA model regarding how the certainty of location information affects children's recall responses. First, according to the model, uncertainty should increase as the length of the delay between viewing and estimating a location increases (Huttenlocher et al., 1991). As fine-grained memory becomes less certain over time, people should rely more heavily on categorical information, thereby exhibiting larger biases toward spatial prototypes and larger response variability (for related ideas, see Engebretson \& Huttenlocher, 1996; Huttenlocher, Hedges, \& Prohaska, 1988; Newcombe et al., 1999). To test these predictions, participants estimated target locations following a short and long delay.

Although we expected response biases to generally increase across delays, we tested a set of more specific predictions in this study. According to the CA model, the increase in error across delays should depend on the geometry of the task space, that is, on the spatial layout of category boundaries and prototypes (Huttenlocher et al., 1991). Thus, we examined delay-dependent effects in four critical spatial regions that varied in "geometric" certainty (see Figure 2). The first region contained locations close to spatial prototypes and far from a midline category boundary (see the circles in Figure 2). Because locations in this region were close to prototypes, we predicted that children's memory for these locations would remain certain over short-term delays, and responses would show little bias and low response variability. Conversely, memory for locations far from spatial prototypes in the second region would be less certain (see the stars in Figure 2). As a consequence, responses to these locations should show large biases toward spatial prototypes over delays. Memory for locations in the third region should also be uncertain because they are far from spatial prototypes (see the diamonds in Figures 2). However, because these locations are also near the category boundary, they can be influenced by an additional source of uncertainty. Specifically, the category membership of locations in this region might be difficult to determine, leading to occasional category membership errors. Such 


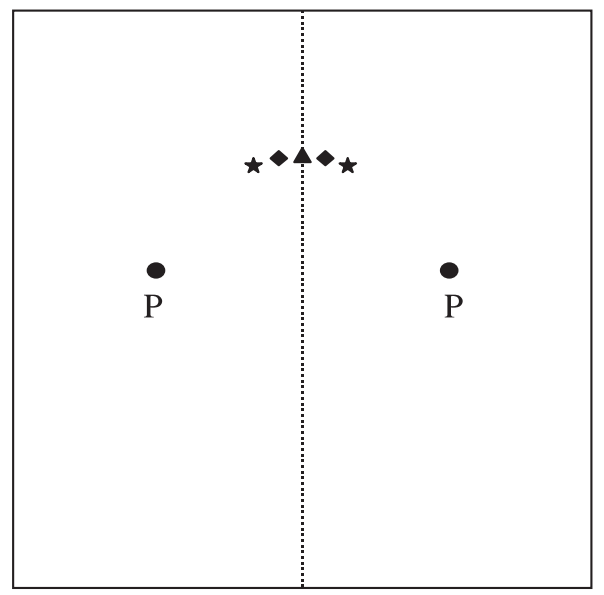

FIGURE 2. Schematic diagram of regions of geometric certainty based on predictions of the CA model. The dashed line marks the midline category boundary, and the Ps mark the location of spatial prototypes. The shapes mark target locations within each of the four regions of certainty. Circles are within the certain region near the prototypes; stars are within the uncertain region far from prototypes; diamonds are within the uncertain region close to the category boundary; the triangle is within the certain region aligned with the midline axis.

errors would increase response variability and could reduce the magnitude of biases toward spatial prototypes over delays. The location in the fourth region shown in Figure 2 is aligned with the midline symmetry axis (see triangle in Figure 2). Thus, memory for this location should be relatively certain over delays, provided that children can accurately perceive this axis.

As a second test of the CA model's claims regarding certainty, we explored how the frequency with which children responded to these locations affected response biases and variability. How might such task-specific experience influence children's memory? One possibility derived from concepts used in the CA model is that frequency will alter the fine-grained certainty of each individual location, thereby affecting the magnitude of categorical bias and response variability in a manner similar to the delay-dependent effects discussed previously. For example, certainty should be high for frequent targets (see Presson, 1987). As such, children should show relatively small categorical biases and low variability when reproducing frequent target locations. Conversely, certainty should be lower for less frequent targets. Thus, children should show larger categorical biases and greater variability when reproducing the location of infrequent targets. As with predictions about uncertainty and delay, frequency-related effects should depend on the geometric certainty of the to-be-remembered locations. In particular, the magnitude of bias should be greatest when the infrequent targets are in uncertain regions of the task space (see Figure 2). 
A second possibility that is more challenging for the CA model is that frequency will influence responses by altering an integrated longer term memory of the possible target locations (Schutte \& Spencer, 2002; Spencer et al., 2001). According to this view, the longer term memory of each individual target location in a task does not simply modulate the fine-grained certainty of one location. Rather, longer term memories of targets that are close to one another can interact with recall responses, causing the longer term memory of one location to influence the recall responses to another location. For instance, Spencer et al. asked 2-year-olds to repeatedly search for a toy hidden at an " $\mathrm{A}$ " location in a large sandbox. After six trials to the A location, the toy was hidden at a "B" location 8 to $10 \mathrm{in}$. from A. Two-year-olds' responses on these B trials were biased toward A. Moreover, these longer term memory biases were built up across trials in the task: Biases toward A were larger after 6 trials to A than after 4 trials to A. Finally, biases toward A depended on the relative locations of A and B with respect to the center of the sandbox (i.e., the spatial prototype). Specifically, the pull toward A was greater when A was closer to the center of the sandbox relative to B than when A was farther from the center relative to B. These results suggest that young children's recall responses are concurrently affected by at least two factors: an integrated longer term memory of locations and spatial prototypes. When these cues cooperate (i.e., when A is in the same direction as the center of the sandbox relative to B), children show large biases toward A. When these cues compete (i.e., when $\mathrm{A}$ is in a direction opposite from the center of the sandbox relative to B), children show smaller biases toward A. Note that children's use of an integrated longer term memory in these studies is conceptually similar to adults" use of "induced" category information in object categorization tasks (Huttenlocher, Hedges, \& Vevea, 2000) in that both concepts refer to an experience-dependent grouping of individual items.

We tested these different views of frequency-related effects across two experiments. In Experiment 1, children recalled the locations of three targets that were close together but spanned a midline category boundary. For instance, in one condition, children responded to the two diamond targets in Figure 2 plus the triangle target that was aligned with the midline axis. In an NB condition, children responded to these three locations equally often. In a bias left (BL) condition, they responded more often to the left target and less often to the center and right targets. With this spatial arrangement of targets, the two views of frequency-related effects make divergent predictions regarding children's response errors to the infrequent right target. If manipulations of response frequency affect fine-grained certainty, then responses to the right target should be biased toward the spatial prototype in the right category (i.e., rightward). Conversely, if children construct an integrated longer term memory of the three locations that is biased toward the most frequent location, then responses to the right target should be biased toward this frequent target (i.e., leftward). 
In Experiment 2, we investigated whether children use an integrated longer term memory of locations when targets are close to a spatial prototype, that is, in regions of the task space where memory should be relatively certain. Children responded to two targets close to the spatial prototype, and one target on the other side of the midline category boundary. As in Experiment 1, participants in the NB condition responded to these three locations equally often. In a bias center (BC) condition, they responded more frequently to the center target than to the left and right targets. If responses to targets close to spatial prototypes can be affected by an integrated longer term memory, then responses to the right target should be biased inward, toward the most frequent target. It is important to note that such biases away from prototypical locations, although challenging, are not precluded by the CA model. Indeed, recent proposals by Huttenlocher et al. (2000) may lay the groundwork for explaining such effects. We return to this issue in the General Discussion.

In addition to testing the CA model by manipulating the certainty of location-related information, a third goal of this study was to examine possible developmental changes in how children weight fine-grained and categorical information. Newcombe and Huttenlocher (2000) proposed that as children become more skilled over development, they are better able to differentially weight location cues in task-specific and flexible ways. If this is the case, younger children might not be able to shift how they combine fine-grained and categorical information as task demands are varied. Consequently, they should show dramatic response errors when location cues are put into conflict, because they cannot resolve this conflict by selecting one cue over others. By contrast, older children may have more advanced location memory skills that allow them to select the most certain information available, thereby minimizing recall errors. Children from two age groups participated in this study: 5- to 6-year-olds and 10- to 11-year-olds. We selected these age groups because previous findings have suggested that children's place-learning abilities change qualitatively between 6 and 10 years of age (Huttenlocher et al., 1994; Sandberg et al., 1996).

\section{EXPERIMENT 1}

\section{Method}

Participants. Twenty-four 6-year-olds $(M=6$ years 5 months; $S D=4.33$ months) and twenty-four 10 - to 11 -year-olds $(M=11$ years 1 month; $S D=6.68$ months) participated in this study. We collected data from 4 additional 6-year-olds; however, these data were not included in the final analyses-one participant stopped data collection early because he did not enjoy the task, and 3 participants were missing more than $8 \%$ of data after initial data analysis (see the information 
that follows). Thirty-six children (twenty 6-year-olds, sixteen 11-year-olds) were recruited from a child participant database maintained by the Department of Psychology at the University of Iowa, and via referrals from other participants. The remaining 16 children (eight 6-year-olds, eight 11-year-olds) were recruited from a child participant database maintained by the Department of Psychology at Indiana University. Children recruited from both communities were predominantly from middle- to upper-middle-class White families. All participants were right-handed. Approximately equal numbers of girls and boys participated in each experimental condition. Children received a $\$ 5$ gift certificate for each session.

Materials. Participants sat at a $1.22-\mathrm{m} \times 1.22-\mathrm{m}$ horizontal table, the top of which was a uniform piece of Plexiglas. An arc was removed from one side of the table, and participants sat in an adjustable chair positioned within this arc with the tabletop at belly height (see Figure 3a). The Plexiglas tabletop was covered with black tinting on one side to prevent participants from seeing the small LEDs positioned below. In addition, the room lights were dimmed and black cloth was hung across the ceiling and down the walls to prevent reflections from appearing on the tabletop. After these adjustments, the top of the table appeared to be a smooth, black, homogeneous surface. A small (1-cm radius) sticker placed at a fixed location from the front edge of the table marked the starting location for each trial.

A moveable panel below the surface of the table included LEDs and an electromagnetic switch. The bank of fixed LEDs with diodes every $10^{\circ}$ from $-90^{\circ}$ to $90^{\circ}$ served as targets. These diodes were located $15 \mathrm{~cm}$ from a starting location. The panel also included a fixation light diode that was $4 \mathrm{~cm}$ in front of the starting location. An X-ray film on top of the LED panel distinguished the target lights from the fixation light: The $\mathrm{X}$-ray film was entirely black except for spaceship shapes (1.25 $\mathrm{cm}$ from tip to base, $1.25 \mathrm{~cm}$ across the base, and $0.65 \mathrm{~cm}$ at the midsection) that were aligned with the target LEDs and a circle shape $(0.5-\mathrm{cm}$ radius) that was aligned with the fixation light (see Figure 3b). An electromagnetic switch was positioned just below the starting location. This was used to ensure that participants were ready to begin each trial, remained at the starting location during the delays, and started moving at the correct time. We positioned the LED panel $15 \mathrm{~cm}$ from the front edge of the table for the 6-year-olds and $20 \mathrm{~cm}$ for the 11 -year-olds.

The lights and switch were controlled by an IBM-compatible computer equipped with an input/output board connected to an electromechanical relay box. The relays were able to trigger the target lights with better than 10-msec precision. The computer controlled the type and timing of all stimuli presented during the experiment using customized software. In addition, the computer monitor presented visual feedback after each trial. We positioned the monitor to the right of the table at a comfortable viewing distance (see Figure 3a). The experimenter sat next to the monitor during data collection sessions. Prerecorded messages were played 
a Optotrak
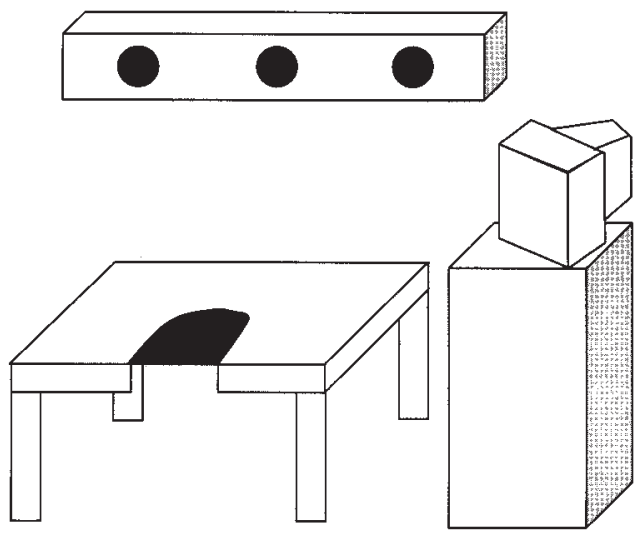

b

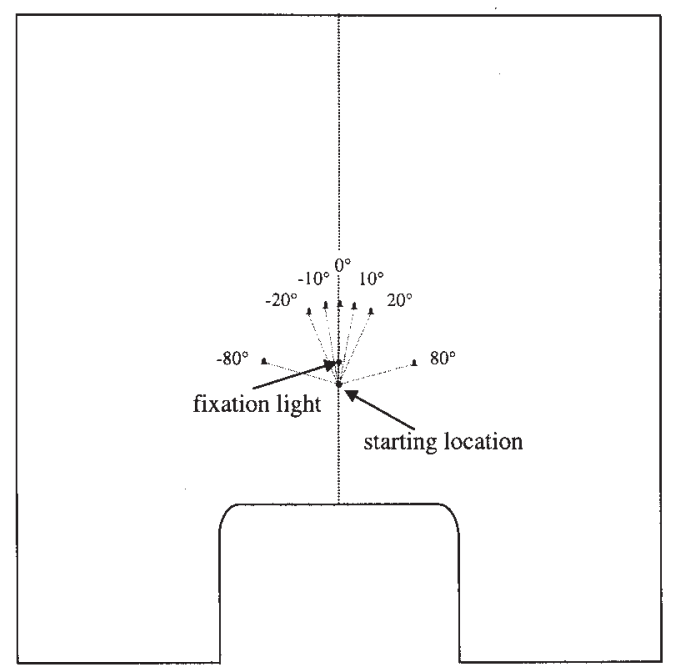

FIGURE 3. (a) Diagram of the experimental table, the Optotrak cameras, and the feedback monitor. (b) Overhead view of tabletop with a diagram of possible target locations (as presented to 11-year-old participants). Dashed line represents the midline symmetry axis.

through two speakers placed on the floor on either side of the table. These messages led participants through the game and gave them feedback on performance.

We recorded participants' movements using an Optotrak optical-electronic motion analysis system (Optotrak 3020, Northern Digital, Inc., Waterloo, Canada). Optotrak tracks small (radius $=3.5 \mathrm{~mm}$ ), individually pulsed infrared emitting diodes (IREDs) within a specified three-dimensional volume, with better than $1 \mathrm{~mm}$ 
precision. IRED data were collected in two dimensions at $150 \mathrm{~Hz}$ and then converted to three-dimensional coordinates using the direct linear transformation technique. Optotrak data were recorded directly to the computer used to control the spaceship lights and participant feedback. Before each data collection session, we collected one trial of Optotrak data from three IREDs embedded in the LED panel. This identified the real-world location of the panel. We placed three IREDs on each participant's right index finger to ensure good IRED visibility. The IRED directly above the fingernail always had the best visibility, so data from this IRED were used in all analyses.

Task and procedure. When participants arrived at the laboratory, they were seated at the experimental table and told that spaceships would appear and then disappear somewhere on the tabletop. Their task was to remember where each spaceship was hiding and to move to the remembered location at the end of a "ready, set, go" sequence spoken by the computer. Participants moved to the target locations by using their right index finger to slide a magnetic disk along the tabletop. The magnet was covered with felt on one side so it would glide easily on the Plexiglas.

After we described the game, parents signed consent forms and a practice session began. During practice, the specific task and types of feedback were explained. Each trial began when the computer said, "Beginning search for enemy spaceships." Then, participants moved the disk to the starting location and attended to the task space on the table in front of them. After a random pretrial delay ranging from 2 to $4 \mathrm{~s}$, a "spaceship" light was illuminated for $2 \mathrm{~s}$ Next, participants heard a "ready, set, go" sequence which ended 0 to $15 \mathrm{~s}$ after the target disappeared. To control participants' looking direction during the delays, a fixation light appeared after the target disappeared. Participants were asked to look at this light rather than look at the location where the spaceship was "hiding." The experimenter made sure that participants looked at the fixation light during each trial. The fixation light was turned off at the start of the "ready, set, go" sequence.

Participants were instructed to move directly to where they thought the spaceship was hiding when they heard "go." Movement speed was not emphasized; however, initiation time to the "go" signal was emphasized. This ensured that the length of the delay periods remained relatively constant across trials and participants. Participants could make small corrections at the end of the movement, but they were asked to maintain a final position until they received feedback from the computer. At the end of each 3.5-s trial, the target was reilluminated for $1.5 \mathrm{~s}(2 \mathrm{~s}$ during the practice phase of Session 1). This allowed participants to compare the location of their finger (the remembered target location) with the actual target location. This type of feedback is similar to that used in other location memory tasks (e.g., Huttenlocher et al., 1994) in which participants see the object emerge from its hiding location at the end of each trial. Feedback information also was dis- 
played on the computer monitor for $3 \mathrm{~s}$ at the end of each trial. Following feedback, the screen was blanked, there was a short delay, and the computer began the next trial. We chose to provide feedback to explore whether helping children monitor their performance would enhance longer term memory effects across trial blocks, and to keep children interested in the task.

Computer feedback on each trial was based on two sources of information. First, the magnetic switch underneath the table was triggered when participants started moving. This allowed the computer to compute an initiation time score. To receive the highest initiation time score (5 points), 6-year-olds had to begin moving within $150 \mathrm{~ms}$ of the "go" signal, and 11-year-olds had to begin moving within $70 \mathrm{~ms}$ of "go." Second, the computer determined the spatial location at the end of each movement using the Optotrak data. These data were used to compute a spatial accuracy score for each trial. Scores ranged from 5 points for movements 0 to 1.5 $\mathrm{cm}$ from the target to 0 points for movements more than $10.5 \mathrm{~cm}$ from the target.

Four types of feedback information were displayed on the computer screen after each trial: (a) the initiation time, (b) the sum of the initiation time and accuracy scores, (c) the total accumulated points, and (d) a "flight rank." The computer warned participants if their initiation times were at the boundaries of the acceptable initiation time range. Point scores of 9 and 10 were accompanied by good job and direct hit! messages, respectively. Participants received one new star for each 80 total points. They heard a verbal message describing their new rank after every two stars earned.

Experimental design. We randomly assigned participants in each age group to one of six experimental conditions in a full factorial design, with bias condition and target separation as between-subject factors. Participants in each condition moved to three target locations - a left, center, and right location. Participants in the BL conditions moved to the left, or biased, target on two thirds of all trials. The remaining trials were divided equally between the nonbiased targets (i.e., the center and right targets). Participants in the NB conditions moved equally often to the left, center, and right targets. In addition, the separation between adjacent targets varied across conditions. In the $10^{\circ}$ conditions, adjacent targets were $10^{\circ}$ apart $\left(-10^{\circ}, 0^{\circ}, 10^{\circ}\right.$; see Figure $\left.3 \mathrm{~b}\right)$; in the $20^{\circ}$ conditions, adjacent targets were $20^{\circ}$ apart $\left(-20^{\circ}, 0^{\circ}, 20^{\circ}\right)$; and in the $80^{\circ}$ conditions, adjacent targets were $80^{\circ}$ apart $\left(-80^{\circ}, 0^{\circ}\right.$, $\left.80^{\circ}\right)$.

Children came into the laboratory for two experimental sessions. Two sessions were required to obtain enough trials to each target at each delay to compute variable errors ( 8 trials), and at the same time keep the total number of trials per session manageable for children. At the start of the first session, participants completed 15 practice trials -5 to each target at randomly selected delays. At the start of the second session, participants completed 6 practice trials -2 to each target at randomly selected delays. Following practice, children in the BL condition completed 56 tri- 
als divided into 4 blocks. There were 20 trials to the left target at each of two delays (D1, D2), 4 trials to the center target at each of two delays, and 4 trials to the right target at each of two delays. Children in the NB condition completed 54 trials divided into 4 blocks. There were 9 trials to each target at each of two delays. For the 6-year-olds, we used delays of $5 \mathrm{~s}$ (D1) and $10 \mathrm{~s}$ (D2). For the 11-year-olds, we used delays of $10 \mathrm{~s}$ (D1) and $15 \mathrm{~s}$ (D2). In addition to these trials, children completed a small number of 0 -sec delay trials during which the target remained illuminated after the "ready, set, go" sequence. There were 3 of these trials to each target during each session. We used these trials to determine how accurate children could be when there were no memory demands. All trials were randomized with the constraint that movements to a nonbiased target never occurred more than twice in a row.

We selected the memory delays and target locations probed in this study based on previous work from our laboratory showing that 6- and 11-year-olds use the midline axis $\left(0^{\circ}\right)$ of our task space to divide the space into two categories with prototypes at $\pm 90^{\circ}$ (Spencer \& Hund, 2002a). In these studies, the apparatus and task were identical to those used here. Children pointed to three locations-a left, center, and right location-following delays of 0 to $20 \mathrm{~s}$. Across conditions, we varied the angular position of the left and right targets relative to midline. The center target was always aligned with the midline of the table (i.e., $0^{\circ}$ ). Responses to the center target were accurate across delays. In contrast, responses to the left and right targets were biased away from midline over delays, and the magnitude of bias depended on the position of each target in the task space. Specifically, biases were greatest for the $\pm 20^{\circ}$ targets and decreased when targets were closer $\left(10^{\circ}\right)$ and farther $\left(40^{\circ}-60^{\circ}\right)$ from midline. Responses to the most extreme targets, $\pm 80^{\circ}$, were relatively accurate over delays. These findings suggest that children used the midline symmetry axis of the table to divide the task space into two regions, and that responses were biased toward spatial prototypes located at $\pm 90^{\circ}$ (for similar results with adults, see Spencer \& Hund, 2002b).

Method of analysis. Three-dimensional motion data were filtered using a second-order recursive low-pass Butterworth filter with a $20-\mathrm{Hz}$ cutoff. The cutoff frequency was determined using the residual analysis proposed by Winter (1990). We used three kinematic events to identify the $x$ and $y$ coordinates at the end of each movement-the end of the "transport" phase, the end of the "correction" phase, and the end of the "extra" correction phase. The computer selected these events using the following rules. The end of the transport phase was defined by one of two possible events: the first tangential velocity minimum or the first data frame $<2 \mathrm{~cm} / \mathrm{s}$ after the velocity dropped below $30 \%$ of the peak velocity and stayed below $30 \%$ for at least one more velocity peak. The end of the correction phase was defined as the first data frame $<2 \mathrm{~cm} / \mathrm{s}$ after the transport phase with a peak correction velocity $>4 \mathrm{~cm} / \mathrm{sec}$ and $<30 \%$ of the peak velocity. The $4 \mathrm{~cm} / \mathrm{s}$ criterion en- 
sured that the computer would search for a velocity peak after the transport phase with a maximum greater than the resting velocity $(2 \mathrm{~cm} / \mathrm{s})$. If no valid velocity peak was found, the computer simply selected the first velocity minimum below the resting velocity. The rules for identifying the correction phase were also used to identify the extra correction phase.

After the computer selected these events, the directional errors for locations at the end of the transport, correction, and extra correction phases were computed. For each endpoint (i.e., $x$ and $y$ coordinate pair), we calculated the angle between the start-end line and the start-target line. Negative directional errors indicate counterclockwise errors relative to the target direction. We used data from the most accurate kinematic event in the final analyses. This was the most conservative choice because it maximized participants' accuracy, which was counter to the goal of investigating increases in memory errors under conditions of uncertainty.

Directional errors were checked for outliers in a three-stage process (for details, see Spencer \& Hund, 2002b). In the first stage, the computer identified all trials in which directional errors were larger than the median error $\pm 2 S D$ s for movements to each target location at each delay. Standard deviations were set to a minimum value of $5^{\circ}$ for very small standard deviations and a maximum value of $10^{\circ}$ for very large standard deviations. Second, these trials were reanalyzed using an interactive version of the automated analysis software to determine whether the large errors were due to computer selection mistakes. Third, using the criterion from the first stage, all remaining trials with large directional errors were eliminated. Trials with initiation times more than $2 \mathrm{~s}$ before or after "go" also were eliminated. Across the entire data set, only one trial did not meet the initiation time criterion. Overall, an average of $3.38 \%$ of all trials were eliminated for the 6-year-olds and $1.41 \%$ for the 11-year-olds.

Finally, we noticed that participants' responses on the 0-s delay trials were rotated slightly, even though the targets were visible. Table 1 shows the mean error to each target location at the 0 -s delay. Inspection of Table 1 reveals that the errors at $0 \mathrm{~s}$ were quite small across targets and experiments. In the final analysis step, we removed these systematic errors by subtracting the mean directional error on the 0 -s delay trials from the directional errors on the short- and long-delay

TABLE 1

Directional Error for Responses to Each

Target at the Zero-Second Delay in Each Experiment

\begin{tabular}{crll}
\hline Experiment & Left target & Center target & Right target \\
\hline 1 & $-1.17^{\circ}\left(.31^{\circ}\right)$ & $-1.38^{\circ}\left(.22^{\circ}\right)$ & $-1.13^{\circ}\left(.26^{\circ}\right)$ \\
2 & $1.10^{\circ}\left(.16^{\circ}\right)$ & $-1.75^{\circ}\left(.23^{\circ}\right)$ & $-1.81^{\circ}\left(.17^{\circ}\right)$ \\
\hline
\end{tabular}

Note. Standard errors are listed in parentheses. 
trials for each target and participant. These adjusted directional errors were used in all analyses.

\section{Results}

As in previous studies which manipulated the angular locations of targets within the task space (e.g., Engebretson \& Huttenlocher, 1996; Sandberg et al., 1996; Schutte \& Spencer, 2002; Spencer \& Hund, 2002b), we analyzed participants' directional errors. Specifically, we computed constant (mean) and variable (standard deviation) directional errors to each target location at each delay. Constant errors reveal categorical biases; variable errors index the stability of memory. We report data from each of these measures in turn.

Constant directional error. Mean constant directional errors were analyzed in a five-way analysis of variance (ANOVA) with condition (BL, NB), age (6 years, 11 years), and separation $\left(10^{\circ}, 20^{\circ}, 80^{\circ}\right)$ as between-subject factors and delay (D1, D2) and target (left, center, right) as within-subjects factors. There was a significant main effect of target, Wilks's $\Lambda=0.24, F(2,35)=56.72, p<.001$, and a significant Target $\times$ Separation interaction, Wilks's $\Lambda=0.45, F(4,70)=8.55, p<$ $.001 .^{1}$ Tests of simple effects indicated that errors across the three target locations differed significantly at $10^{\circ}, F(2,72)=38.60, p<.01$, and at $20^{\circ}, F(2,72)=68.97$, $p<.01$, but not at $80^{\circ}, F(2,72)=1.79, n s$. This interaction is evident in Figure 4 . Children generally made large outward errors-errors toward the spatial prototypes-when moving to the left and right targets, but only when the targets were far from spatial prototypes at $90^{\circ}\left(10^{\circ}\right.$ and $20^{\circ}$, see upper and middle panels of Figure 4). Close to spatial prototypes (i.e., $80^{\circ}$ ), in contrast, children's constant errors to the left and right targets were very small (see bottom panels of Figure 4).

There was also a significant Delay $\times$ Target interaction, Wilks's $\Lambda=0.70, F(2$, $35)=7.61, p<.005$. Tests of simple effects indicated that children's directional errors increased significantly over delays when they moved to the left target, $F(2,72)$ $=38.60, p<.01$ (D1: $M=-3.33^{\circ}, S D=2.86$; D2: $M=-3.98^{\circ}, S D=3.63$ ), and the right target, $F(2,72)=38.60, p<.01\left(\mathrm{D} 1: M=2.10^{\circ}, S D=3.64 ; \mathrm{D} 2: M=2.83^{\circ}, S D\right.$ $=4.30)$, but not when they moved to the center target, $F(2,72)=38.60, n s(\mathrm{D} 1: M=$ $-1.14^{\circ}, S D=2.45 ; \mathrm{D} 2: M=-.82^{\circ}, S D=2.82$ ). Thus, as predicted by the CA model, errors to targets within the left and right categories increased significantly over delay.

Finally, there was a significant Age $\times$ Condition interaction, $F(1,36)=9.32, p<$ .005 . Tests of simple effects indicated that these directional errors differed signifi-

\footnotetext{
${ }^{1}$ We used multivariate tests of within-subjects factors (Wilkes $\Lambda$ ) in all analyses because these tests do not require the assumption of sphericity. Thus, they are more conservative than conventional univariate tests of within-subjects factors.
} 


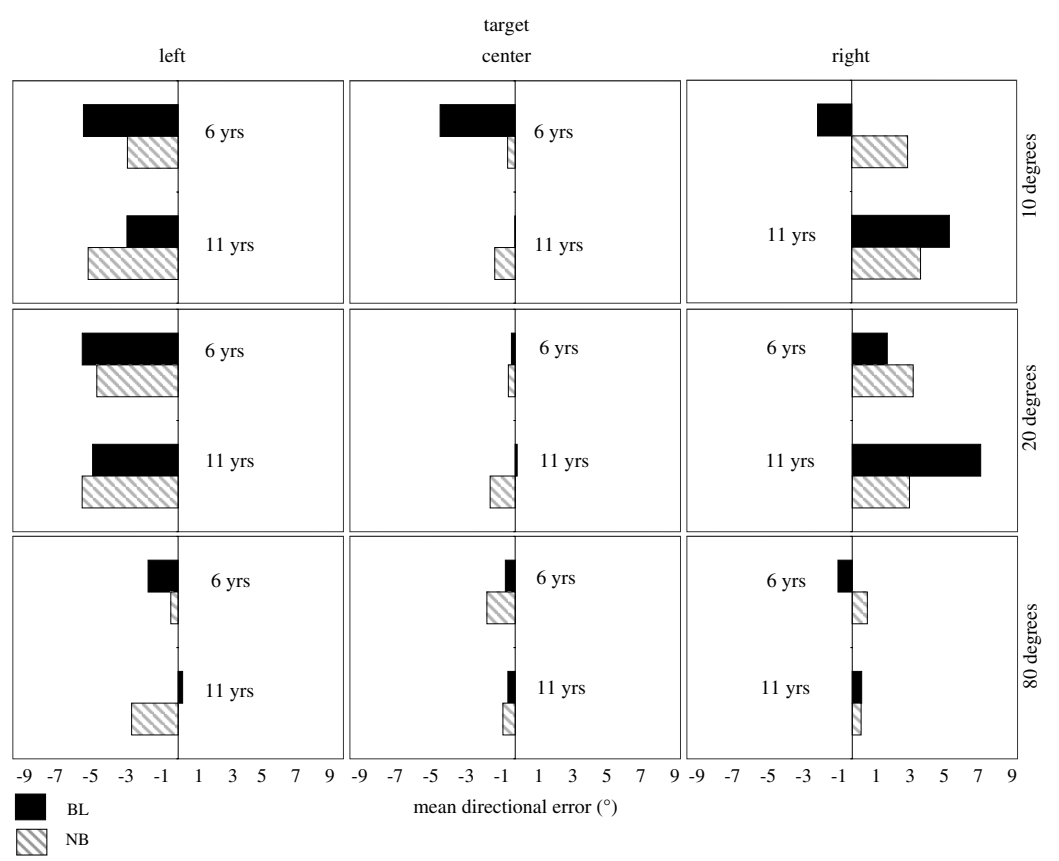

FIGURE 4. Mean constant directional errors in Experiment 1 to the left (left panels), center (center panels), and right (right panels) targets for each condition across the $10^{\circ}$ (top panels), $20^{\circ}$ (center panels), and $80^{\circ}$ (bottom panels) target separations for each age. Striped bars show constant errors in the NB condition; solid bars show constant errors in the BL condition. Positive errors reflect clockwise errors; negative errors reflect counterclockwise errors.

cantly from errors in the NB condition for each age group: 6-year-olds, $F(1,36)=$ $5.01, p<.05$, and 11-year-olds, $F(1,36)=4.32, p<.05$. However, the direction of error in the BL condition differed across age groups. That is, 6-year-olds made larger leftward errors in the BL condition $\left(M=-2.17^{\circ}, S D=3.18\right)$ than in the NB condition $\left(M=-.34^{\circ}, S D=1.00\right)$, whereas 11-year-olds made rightward errors in the BL condition $\left(M=.66^{\circ}, S D=1.74\right)$ and leftward errors in the NB condition $(M$ $\left.=-1.04^{\circ}, S D=1.15\right)$. Inspection of Figure 4 suggests that these differences in the $\mathrm{BL}$ condition were due to a reduction in rightward error (or slight leftward error) to the infrequent right target for 6-year-olds and an increase in rightward error (e.g., toward the spatial prototype) to the infrequent right target for 11-year-olds. No other effects from the overall ANOVA reached significance.

Given the lack of significant target effects in the $80^{\circ}$-separation condition (when the targets were close to spatial prototypes) and the small errors depicted in Figure 4, we conducted a follow-up analysis to determine whether there were any 
significant delay or bias-related effects at the $80^{\circ}$-target separation. Specifically, mean constant directional errors from the $80^{\circ}$ separation were entered into a Condition $\times$ Age $\times$ Delay $\times$ Target ANOVA. There were no significant effects in this analysis. Thus, children's responses to targets close to spatial prototypes did not change over delay or as the frequency of presentation was varied.

Analyses of learning effects. If children's ability to remember location information is affected by an integrated longer term memory of the target locations - as the significant bias-related effects for the 6-year-olds suggest - then the build up of longer term memory effects should be evident across trials. To investigate this possibility, we analyzed children's errors to the left and right targets in the $10^{\circ}$ and $20^{\circ}$ conditions across four blocks of trials: early during Session 1 (Block 1), late during Session 1 (Block 2), early during Session 2 (Block 3), and late during Session 2 (Block 4). Data from the center target and from the $80^{\circ}$ condition were excluded from this analysis because children exhibited very small errors in these cases and showed no significant bias-related effects. Trial 32 divided the early and late learning blocks in each session. This trial was nearly halfway through the session regardless of condition or session number. Due to the randomization of trial order, there were some cases in which children did not move to the left or right target at a particular delay during one of the learning blocks. Thus, we collapsed across the short and long delays, computing the median directional error to the left and right targets in each trial block. Median errors were used because of the small number of trials in some blocks.

Median directional errors were analyzed in a five-way ANOVA with condition (BL, NB), age (6 years, 11 years), and separation $\left(10^{\circ}, 20^{\circ}\right)$ as between-subject factors and block $(1,2,3,4)$ and target (left, right) as within-subjects factors. Only block effects are reported in the information that follows. There was a significant Block $\times$ Target interaction, Wilks's $\Lambda=0.63, F(3,22)=4.38, p<.05$, and a significant Block $\times$ Target $\times$ Age $\times$ Condition interaction, Wilks's $\Lambda=0.71, F(3,22)=$ $3.04, p<.05$. Tests of simple effects revealed a significant block effect for 6-year-olds in the BL condition, $F(3,72)=3.84, p<.05$, as well as a significant Block $\times$ Target interaction in this condition, $F(3,72)=5.35, p<.05$. As can be seen in Figure 5, these differences were caused by a shift in the direction of response bias to the right target in this condition-toward the spatial prototype (i.e., rightward) in Block 1 and toward the biased target (i.e., leftward) in Blocks 2 to 4 . This suggests that as 6-year-olds' longer term memory of the frequent target became stronger, their responses to the infrequent target were pulled toward the biased target in the opposite category. Inspection of the data from individual 6-year-olds revealed that this inward bias was not driven by only 1 or 2 children, but was representative of the group. That is, six of eight 6-year-olds exhibited counterclockwise errors (i.e., toward the biased target). Additional tests of simple effects revealed a significant Block $\times$ Target interaction for the 11-year-olds, $F(3,72)=2.79, p<.05$. 


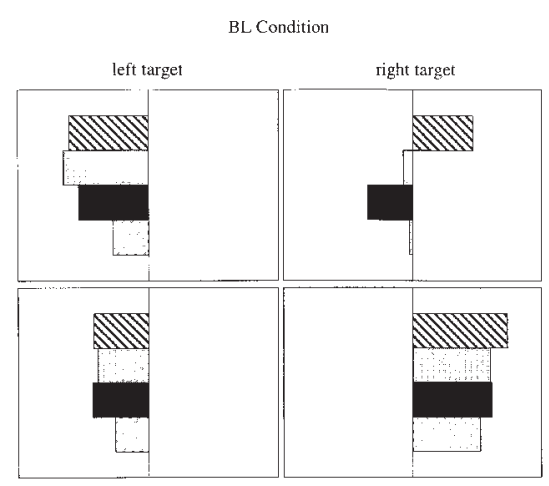

$10-8 \quad-6 \begin{array}{ccccccccccccccccccc}-4 & -2 & 0 & 2 & 4 & 6 & 8 & 10 & -10 & -8 & -6 & -4 & -2 & 0 & 2 & 4 & 6 & 8 & 10\end{array}$ median directional error $\left({ }^{\circ}\right)$

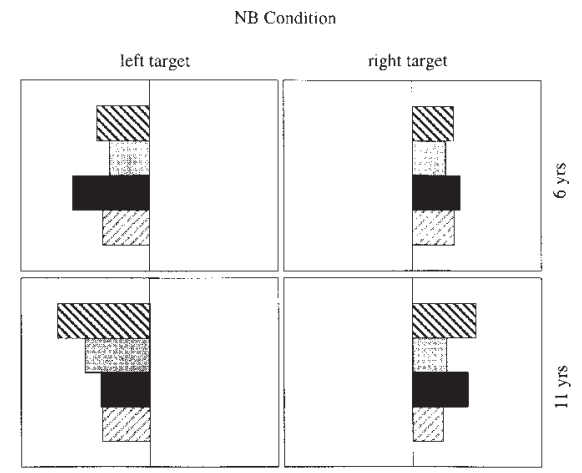

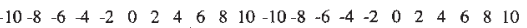
median directional error $\left({ }^{\circ}\right)$

Block 1

Block 2

Block 3

Bluck 4

FIGURE 5. Median directional errors in Experiment 1 to the left and right targets for 6-year-olds (top panels) and 11-year-olds (bottom panels) in the BL (left panels) and NB (right panels) conditions. Different bar styles show errors across the four blocks of trials.

In contrast to the 6-year-olds, this interaction occurred in the NB condition. As can be seen in Figure 5, 11-year-olds' performance to the left target in this condition showed a large improvement over blocks.

Variable directional error. Children's mean variable directional errors were entered into a five-way ANOVA with condition (BL, NB), age (6 years, 11 years), and separation $\left(10^{\circ}, 20^{\circ}, 80^{\circ}\right)$ as between-subject factors and delay (D1, D2) and target (left, center, right) as within-subjects factors. There were significant main effects of delay, Wilks's $\Lambda=0.66, F(1,36)=18.50, p<.001$, target, Wilks's $\Lambda=$ $0.38, F(2,35)=28.06, p<.001$, and age, $F(1,36)=25.12, p<.001$. These main effects were subsumed by a Delay $\times$ Target $\times$ Age interaction, Wilks's $\Lambda=0.82, F(2$, $35)=3.83, p<.05$. Tests of simple effects indicated that responses to the left and right targets were more variable than were responses to the center for both 6-year-olds, $F(2,46)=12.24, p<.001$, and 11-year-olds, $F(2,46)=22.81, p<.001$ (see the V-shaped pattern evident in most panels in Figure 6). In addition, variability increased significantly over delays for both age groups: 6-year-olds, $F(1,23)=$ $8.53, p<.01$ (D1: $M=4.35^{\circ}, S D=1.30 ; \mathrm{D} 2: M=5.00^{\circ}, S D=1.58$ ), and 11 -year-olds, $F(1,23)=10.85, p<.005$ (D1: $M=3.36^{\circ}, S D=.63$; D2: $M=3.73^{\circ}$, 
$S D=.82$ ). The increase in variability over delays for the 11-year-olds was most dramatic to the left target.

The five-way ANOVA also revealed a significant separation main effect, $F(2$, $36)=8.64, p<.001$. Children's responses were more variable at the $10^{\circ}$ separation $\left(M=4.73^{\circ}, S D=1.48\right)$ than at the $20^{\circ}\left(M=3.99^{\circ}, S D=.99\right)$ and $80^{\circ}$ separations $(M$ $\left.=3.61^{\circ}, S D=.78\right)$. Finally, there was a significant condition main effect, $F(1,36)=$ 7.94, $p<.01$, and a significant Age $\times$ Condition interaction, $F(1,36)=8.72, p<$ .01 . Tests of simple effects revealed a significant difference in variability across conditions for the 6-year-olds, $F(1,36)=16.64, p<.01$, but not for the 11 -year-olds, $F(1,36)=0.009$, ns. Six-year-olds' responses were more variable in the BL condition than in the NB condition (see Figure 6). This was particularly evi-

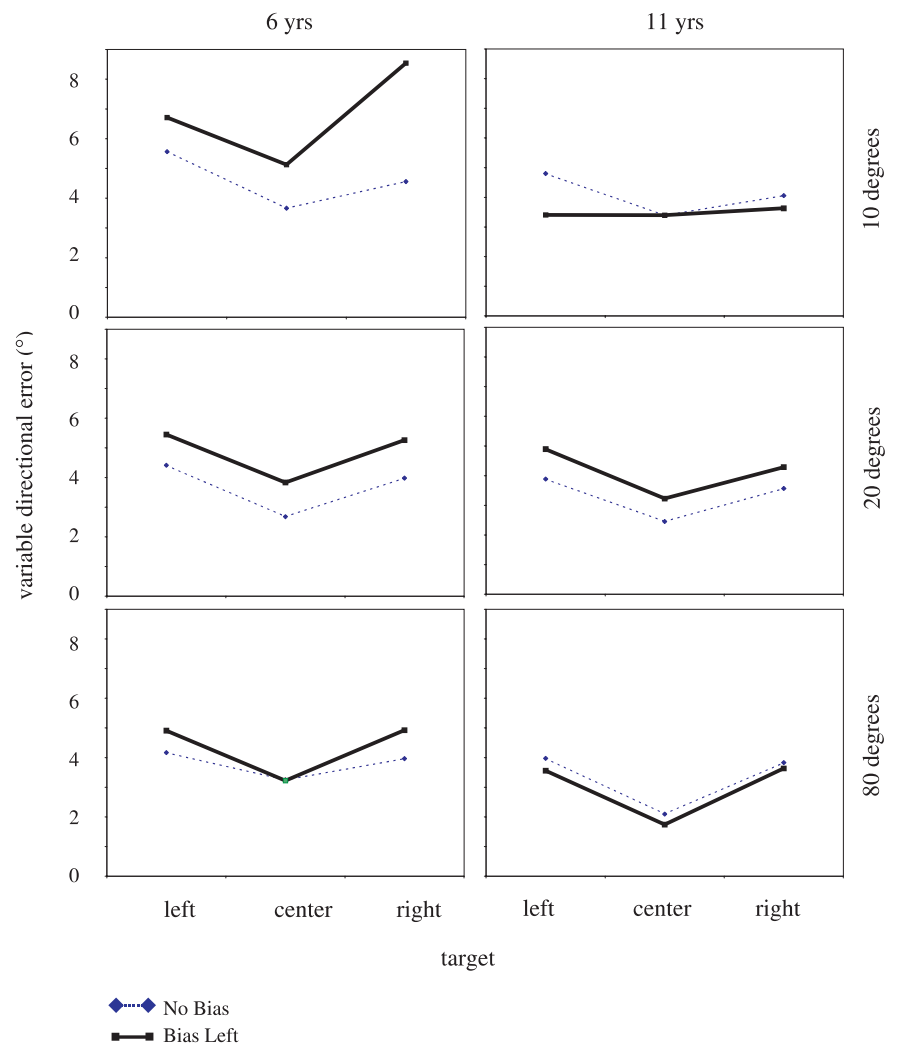

FIGURE 6. Mean variable (standard deviation) directional errors in Experiment 1 to the left, center, and right targets for each condition across the $10^{\circ}$ (top panels), $20^{\circ}$ (middle panels), and $80^{\circ}$ (bottom panels) target separations for the 6-year-olds (left panels) and 11-year-olds (right panels). Dotted lines show variable errors in the No Bias condition; solid lines show variable errors in the Bias Left condition. 
dent for movements to the infrequent right target in the $10^{\circ}$ condition. Eleven-year-olds, in contrast, showed comparable variability across the BL and NB conditions. No other effects from the ANOVA reached significance.

As with constant error, we conducted a follow-up analysis to determine whether there were any significant delay- or bias-related variable error effects at the $80^{\circ}$ target separation (i.e., close to the prototypes). Mean variable directional errors from the $80^{\circ}$ separation were entered into a Condition $\times$ Age $\times$ Delay $\times$ Target ANOVA. Results revealed significant main effects of target, Wilks's $\Lambda=$ $0.21, F(2,11)=20.59, p<.001$, and age, $F(1,12)=8.91, p<.05$. However, there were no significant bias condition or delay effects. Thus, once again, when targets were close to spatial prototypes, the delay and frequency manipulations had no effect.

\section{Discussion}

The goal of this experiment was to test two predictions of the CA model concerning how certainty influences children's location memory responses. First, we tested whether children's response biases and variability increased across delays. As predicted, constant and variable errors increased significantly between the short and long delays. Moreover, these delay-dependent effects were most dramatic for targets in uncertain regions of the task space (far from spatial prototypes) and least dramatic for targets in certain regions of the task space (close to spatial prototypes or aligned with the category boundary). Thus, consistent with the CA model, children's responses were more strongly biased toward spatial prototypes under conditions of uncertainty-following a long delay in uncertain regions of the task space.

In a second test, we explored whether the frequency with which children responded to each target location influenced location recall. Of central interest was whether children's responses to the infrequent right target would show biases toward spatial prototypes or toward the biased target. As with the delay-dependent effects, frequency affected children's responses only to targets in uncertain regions of the task space for both age groups. However, there were developmental differences in how the frequency manipulation affected children's responses. For the 11-year-olds, frequency effects were consistent with the fine-grained certainty view. That is, when 11-year-olds responded to the right target in the BL condition, they made larger errors toward spatial prototypes. Thus, less frequent experience responding to the nonbiased location reduced the certainty of this location in memory, and 11-year-olds relied more heavily on categorical information. In contrast, repeated experience moving to the biased location enhanced the certainty of this location in memory (see also Presson, 1987), and 11-year-olds relied less on categorical information when reproducing the left location. 
Six-year-olds also showed experience-dependent effects when the targets were in uncertain regions of the task space. Unlike the 11-year-olds, however, 6-year-olds showed a reduction in prototypical biases when responding to the infrequent right target, particularly in the $10^{\circ}$ condition. Moreover, the variability of these responses was quite high. Analyses of learning effects clarified the origin of these effects. Six-year-olds initially showed biases toward the spatial prototypes; however, after several blocks of trials, responses reversed direction and were pulled toward the biased target. These findings are consistent with the integrated longer term memory view of frequency-related effects (see Schutte \& Spencer, 2002; Spencer et al., 2001). Although it is possible that these developmental differences resulted from anomalous effects caused by our relatively small sample size, we do not believe that this is the case given that most of the children in our sample showed inward biases. Moreover, we have observed robust effects across a number of studies using similar designs and sample sizes (e.g., Spencer \& Hund, 2002a, 2002b).

The most surprising characteristic of the 6-year-olds' results is that the frequency-related effects spanned across a category boundary. According to the CA model, the category membership of a location is determined at target presentation - the child sees a target and encodes its fine-grained and categorical location. These two types of information are then combined at recall to generate a response. From this perspective, locations in one category should have no influence on the memory for locations in an adjacent category. Six-year-olds' responses apparently violated this principle. We investigated whether 6-year-olds frequently miscategorized the right target. There was little evidence to support this possibility. Although 6-year-olds occasionally made responses on the wrong side of midline, more often, they placed the right target on the correct side of midline, just closer to this axis than it actually was. Another possibility is that 6-year-olds' responses were not cross-category errors at all. Rather, 6-year-olds flexibly changed how they categorized the task space over learning. Across blocks of trials, for example, 6-year-olds might have started grouping all three targets into one category. Although this could explain why the infrequent, right target was biased inward, this view does not explain why the frequent, left target was consistently biased outward toward the left prototype, nor does it explain why children's performance to the center target was generally accurate. Thus, results suggest that two factors influenced 6-year-olds' responses: spatial prototypes and an integrated longer term memory of locations.

Interestingly, we did not find significant frequency-related effects in the $80^{\circ}$ condition for either age group. There are two possible explanations for this. According to the CA model, manipulations of target frequency should not be effective at the $80^{\circ}$ locations because these locations are close to spatial prototypes in a region of relative certainty. It is also possible, however, that children's accurate performance at $80^{\circ} \mathrm{re-}$ sulted from the large spatial separation between adjacent targets. That is, children's 
responses were accurate to the $\pm 80^{\circ}$ targets, not because these targets were in regions of certainty, but because they were far enough away from the target at $0^{\circ}$ that the longer term memories associated with these locations did not interact. If this is the case, then it should be possible to pull 6-year-olds' responses to the $80^{\circ}$ location away from the spatial prototype in the same manner in which we pulled their responses away from prototypes in this experiment - by placing a biased target nearby. Experiment 2 tested this possibility. Moreover, this experiment explored whether the developmental differences evident in Experiment 1 generalized to conditions in which a biased target was close to a spatial prototype in a region of geometric certainty.

\section{EXPERIMENT 2}

There were three primary goals of this experiment. The first goal was to examine whether frequency-related effects influence children's responses to targets near spatial prototypes (e.g., 90 $)$. Therefore, children responded to two targets that were close to a spatial prototype in a region of relative certainty $-70^{\circ}$ and $80^{\circ}$. We used the $80^{\circ}$ target because children's responses to this location in Experiment 1 were consistently accurate. The second target was positioned $10^{\circ}$ away from the first in the direction opposite from the spatial prototype. We selected a $10^{\circ}$ separation because the frequency-related effects in Experiment 1 were most dramatic at this separation. Children in an NB condition moved to these locations equally often. Children in a bias condition moved to the $70^{\circ}$ location more frequently. If children's recall responses are affected by an integrated longer term memory of the target locations, then responses to the infrequent right target $\left(80^{\circ}\right)$ in the bias condition should be pulled toward the most frequent target $\left(70^{\circ}\right)$ and away from the spatial prototype. Conversely, if children's memory for locations is relatively certain near spatial prototypes, then responses to the infrequent right target should be accurate.

The second goal of this experiment was to reexamine frequency-related effects in an uncertain region of the task space. In Experiment 1, 11-year-olds' responses to infrequent targets in uncertain spatial regions were biased toward the spatial prototype; however, 6-year-olds' responses were biased toward the frequent target (in the opposite direction). As discussed previously, this developmental difference likely reflects 6-year-olds' use of an integrated longer term memory of the target locations. Interestingly, however, 6-year-olds did appear to use information about spatial prototypes - responses to the left and right targets in the $10^{\circ}$ and $20^{\circ} \mathrm{NB}$ conditions were biased toward prototypical locations, as were responses to the infrequent right location in the first block of trials. This raises an important question: Are there conditions in which 6-year-olds will show larger biases toward spatial prototypes when recalling the location of an infrequent target? 
We investigated this possibility by including a third target in this experiment-a target at $-10^{\circ}$. This target was $80^{\circ}$ away from the closest target $\left(70^{\circ}\right)$ - the same spatial separation at which we failed to find frequency-related effects in Experiment 1-but within an uncertain region of the task space. Thus, interactions between the longer term memory of this location and the other two locations should be minimal, and geometric uncertainty should be maximal. Children responded to the $-10^{\circ}$ location as often as to the other locations in the NB condition, and infrequently in the bias condition. According to the CA model, children should show larger biases toward the spatial prototype at $-90^{\circ}$ when responding to the $-10^{\circ}$ target in the bias condition relative to performance in the NB condition. The critical question was whether this would be the case for both age groups.

The third goal of this study was to provide a test for replication of the delay-dependent effects from Experiment 1. Thus, children responded to targets following short and long delays. According to the CA model, children's responses to targets in an uncertain region of the task space should become increasingly biased during delays. By contrast, responses to targets in a certain region of the task space should be relatively accurate over delays.

\section{Method}

Participants. Twelve 6-year-olds $(M=6$ years 6.33 months; $S D=0.49$ months) and twelve 10- to 11 -year-olds ( $M=11$ years 2.0 months; $S D=1.65$ months) participated in this study. Data from 2 additional 6-year-olds were not included in the final analyses because the participants were missing more than $8 \%$ of data following initial data analysis. Children were recruited from a participant database maintained by the Department of Psychology at the University of Iowa. Virtually all participants were right-handed. One 6-year-old reported being ambidextrous, although he used his right hand during the experimental sessions. The number of girls and boys was roughly balanced across experimental conditions. Children received a $\$ 4$ gift certificate for each session.

Materials, task, and procedure. Although the apparatus was nearly identical from the participants' perspective, several modifications made the apparatus more functional for the experimenters. First, the table size was increased to $1.22 \mathrm{~m}$ $\times 1.83 \mathrm{~m}$, and the Plexiglas top was replaced by a rear-projection surface. Next, a Barco 708 Data Projector (Barco, Inc., Belgium) was used to project images onto the table's surface. These images - a yellow start disc, a white fixation disc, and the target spaceships-were similar in size and luminance to those used in Experiment 1. Third, real-time Optotrak analyses of participants' finger position replaced the electromagnetic switch. Thus, Optotrak data were used to ensure that partici- 
pants were ready to begin each trial, remained at the starting location during the delays, and started moving at the correct time. Finally, feedback was projected onto the table's surface following each trial, rather than being displayed on an external monitor. The task and procedure were identical to those used in the previous experiment.

Experimental design. Participants from each age group were randomly assigned to one of two experimental conditions. In each condition, participants moved to three target locations: $-10^{\circ}, 70^{\circ}$, and $80^{\circ}$. As in Experiment 1, participants in an NB condition moved equally often to the three targets. Participants in a bias center (BC) condition moved to the biased target (i.e., $70^{\circ}$ ) on two thirds of all trials. The remaining trials were divided equally between the nonbiased targets.

Method of analysis. The method of analysis was identical to the previous experiment. As in Experiment 1, we removed systematic errors evident in the 0 -sec delay trials from the directional errors on the short- and long-delay trials (see Table 1). These adjusted directional errors were used in all analyses. No trials exceeded the 2-sec initiation time criterion. As a result of the outlier analysis, an average of $4.96 \%$ of all trials were eliminated for the 6 -year-olds and $1.44 \%$ of all trials for the 11-year-olds.

\section{Results}

Constant directional error. Mean constant directional errors were analyzed in a four-way ANOVA with condition (BC, NB) and age (6 years, 11 years) as between-subject factors and delay (D1, D2) and target (left, center, right) as within-subjects factors. There was a significant main effect of delay, Wilks's $\Lambda=$ $0.05, F(1,20)=5.63, p<.05$. As in the previous experiment, directional errors increased significantly over delays (D1: $M=-1.27^{\circ}, S D=1.27 ; \mathrm{D} 2: M=-1.81^{\circ}, S D$ $=1.64)$. There was also a significant main effect of target, Wilks's $\Lambda=0.17, F(2$, $19)=45.57, p<.001$. As can be seen in Figure 7, children made large outward errors (i.e., toward the prototype) to the left target $\left(-10^{\circ}\right)$, which was close to the category boundary in an uncertain region of the task space, and small errors to the center and right targets $\left(70^{\circ}, 80^{\circ}\right)$, which were close to the spatial prototype in a certain region of the task space. Finally, there was a significant Target $\times$ Age $\times$ Condition interaction, Wilks's $\Lambda=0.70, F(2,19)=4.12, p<.05$. Tests of simple effects indicated that the $\mathrm{BC}$ and NB conditions differed significantly at the $-10^{\circ}$ target for 11-year-olds, $F(1,10)=9.70, p<.025$, but not for the 6-year-olds, $F(1,10)$ $=1.81$, ns. Consistent with the CA model, 11-year-olds in the bias condition made larger outward errors (i.e., toward the prototype) to the left target than did 


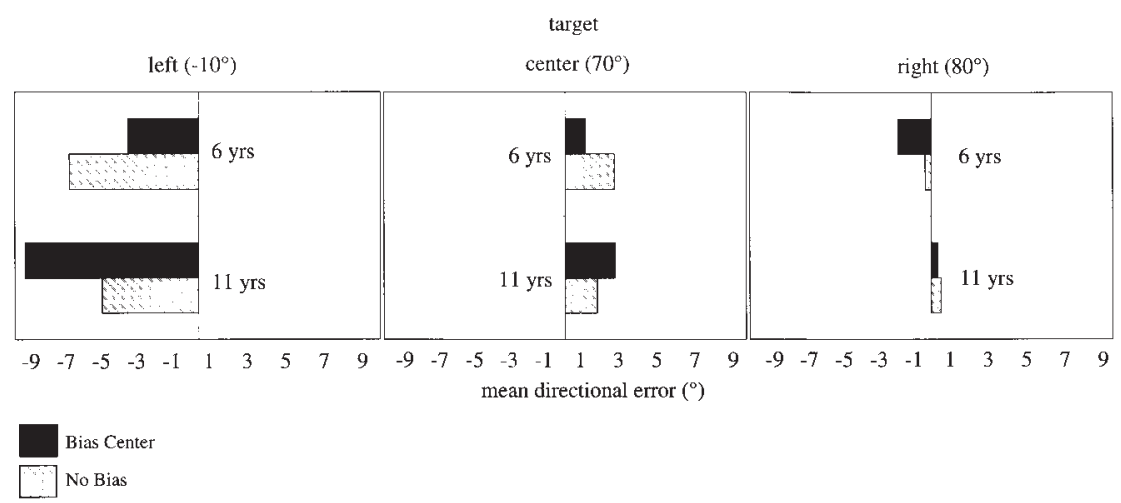

FIGURE 7. Mean constant directional errors to the left (left panel), center (center panel), and right (right panel) targets for participants in the Bias Center (solid bars) and No Bias (striped bars) conditions from Experiment 2.

11-year-olds in the NB condition (see Figure 7). There were no other significant bias-related effects for either age.

Analyses of learning effects. As in Experiment 1, we investigated whether there were significant changes in bias toward the most frequent target across blocks of trials. Median directional errors were analyzed in a four-way ANOVA with age (6 years, 11 years) and condition (BC, NB) as between-subject factors and block $(1,2,3,4)$ and target (left, right) as within-subjects factors. Only block effects are reported. There was a significant Block $\times$ Target $\times$ Condition interaction, Wilks's $\Lambda=0.56, F(3,18)=4.64, p<.05$. Tests of simple effects revealed a significant block effect in the NB condition, $F(3,60)=3.88, p<.05$, and a significant Block $\times$ Target interaction in the $\mathrm{BC}$ condition, $F(3,60)=3.96, p<.05$. Figure 8 shows median directional errors across blocks to the left and right targets in the $\mathrm{NB}$ and $\mathrm{BC}$ conditions. Directional errors generally decreased across blocks in the NB condition, particularly for responses to the left target. As in the previous experiment, this suggests that children benefited from repeated experience in the task. Directional errors to the left target in the $\mathrm{BC}$ condition also tended to decrease across Blocks 2 to 4 . However, errors to the right target $\left(80^{\circ}\right)$ in the $\mathrm{BC}$ condition increased in Block 4. More important, this increase in inward error was in the direction of the biased target $\left(70^{\circ}\right)$ and away from the spatial prototype $\left(90^{\circ}\right)$. These data are consistent with the notion that children's responses are affected by an integrated longer term memory of the target locations, even within a relatively certain region of the task space. 


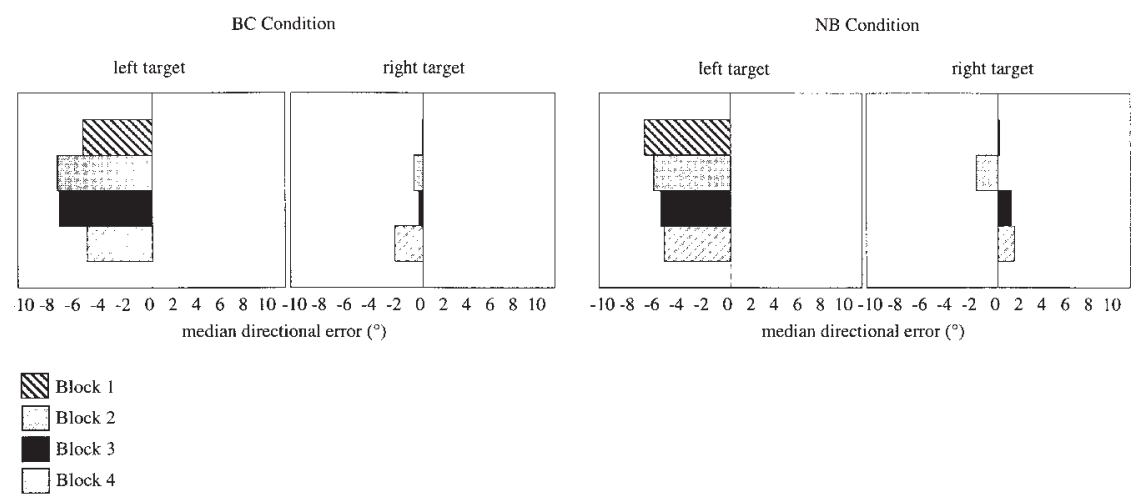

FIGURE 8. Median directional error in Experiment 2 to the left and right targets in the BC (left panels) and NB (right panels) conditions across the four blocks of trials.

Cross-experiment comparison. The two sets of constant error analyses previously mentioned produced mixed results. Analyses of mean directional error showed significant frequency-related effects at the $-10^{\circ}$ location for the 11-year-olds, but no significant effects at the $80^{\circ}$ location for either age group. By contrast, analyses of median directional error over learning showed a significant reduction in error to the $-10^{\circ}$ location and an increase in error toward the biased target to the $80^{\circ}$ location. Thus, we conducted a follow-up analysis to investigate mean directional errors to the $80^{\circ}$ location in greater detail. Specifically, we compared responses to the $80^{\circ}$ target in this experiment, in which the nearest target was $10^{\circ}$ away, with responses to the same spatial location in Experiment 1, in which the nearest target was $80^{\circ}$ away.

Mean constant directional errors to the $80^{\circ}$ target from Experiments 1 and 2 were analyzed in a four-way ANOVA with condition (bias, NB), age (6 years, 11 years), and experiment $(1,2)$ as between-subject factors and delay (D1, D2) as a within-subjects factor. Results showed a significant Delay $\times$ Experiment interaction, Wilks's $\Lambda$ $=0.83, F(1,32)=6.78, p<.025$, and a significant Delay $\times$ Condition $\times$ Experiment interaction, Wilks's $\Lambda=0.85, F(1,32)=5.83, p<.05$. Tests of simple effects revealed a significant Delay $\times$ Experiment effect in the bias condition, $F(1,32)=12.59, p<$ .025 . Additional tests of simple effects indicated that responses in the bias condition differed across experiments at the long delay, $F(1,32)=14.01, p<.025$, but not at the short delay, $F(1,32)=1.62, n s$. At the long delay in the bias conditions, participants made inward errors when a biased target was nearby (Experiment 2: $M=-1.23^{\circ}, S D$ $=2.22$ ) and outward errors when the other targets were far away (Experiment 1: $M=$ $\left..56^{\circ}, S D=2.98\right)$. Participants in both experiments made outward errors in the NB condition (Experiment 1: $M=.84^{\circ}, S D=1.33$; Experiment $2: M=.17^{\circ}, S D=1.79$ ). 
These inward errors in the bias condition of Experiment 2 provide converging evidence that task-specific experience can influence responses to targets within certain regions of the task space.

Variable directional error. Mean variable directional errors were analyzed in a four-way ANOVA with condition (BC, NB) and age as between-subject factors and delay and target as within-subjects factors. There was a significant main effect of delay, Wilks's $\Lambda=0.55, F(1,20)=16.71, p<.005$, and a significant Delay $\times$ Target interaction, Wilks's $\Lambda=0.65, F(2,19)=5.21, p<.05$. Tests of simple effects revealed that variable errors increased significantly across the short and long delays for the left target, $F(1,23)=22.04, p<.001$ (D1: $M=3.68^{\circ}, S D=1.28$; D2: $\left.M=4.93^{\circ}, S D=1.54\right)$, but not for the center target, $F(1,23)=0.97$, ns (D1: $M=$ $4.15^{\circ}, S D=.92$; D2: $\left.M=4.34^{\circ}, S D=1.23\right)$, or the right target, $F(1,23)=0.36, n s$ (D1: $M=3.88^{\circ}, S D=.89 ; \mathrm{D} 2: M=4.04^{\circ}, S D=1.32$ ). This delay-dependent increase in variability across delays for the left target - the target close to the category boundary in an uncertain region in the task space-replicates findings from Experiment 1. Moreover, the absence of delay-dependent effects for the targets close to the prototype $\left(70^{\circ}, 80^{\circ}\right)$ is consistent with results at $80^{\circ}$ in Experiment 1 , suggesting that delay-dependent effects on variability are relatively small near spatial prototypes. In contrast to results from Experiment 1, the variability of 6-year-olds' responses in the $\mathrm{BC}$ and $\mathrm{NB}$ conditions did not differ significantly (see Figure 9). Moreover, variability was generally comparable for both age groups. Of particular note, the $\mathrm{V}$-shaped pattern prevalent in the previous experi-

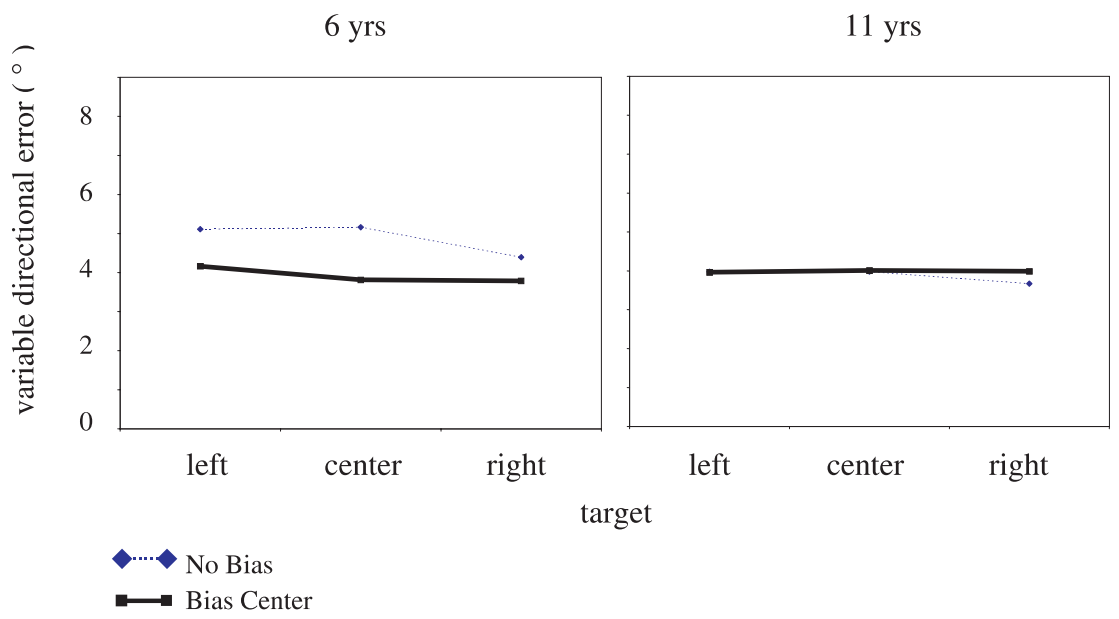

FIGURE 9. Mean variable (standard deviation) directional errors to the left, center, and right targets in Experiment 2 for the 6-year-olds (left panel) and 11-year-olds (right panel) in the Bias Center (solid lines) and No Bias (dashed lines) conditions. 
ment is not apparent in Figure 9. That $\mathrm{V}$ shape resulted from low variability to the center target - the target aligned with the midline category boundary. Thus, the flat variability in this experiment likely reflects the absence of a target at $0^{\circ}$.

\section{Discussion}

One goal of this experiment was to provide a test for replication of the delay-dependent effects reported previously. As in Experiment 1, constant errors increased significantly over delays, as did variable errors to the $-10^{\circ}$ target. Moreover, children's errors varied systematically with the geometric certainty of each location. Children made errors toward a spatial prototype when responding to a target close to the midline category boundary $\left(-10^{\circ}\right)$, and were relatively accurate when responding to targets far from the category boundary $\left(70^{\circ}, 80^{\circ}\right)$.

A second goal of this experiment was to determine whether the responses of children in both age groups would show increases in bias toward the prototype when they responded to an isolated target in an uncertain region of the task space. As in the first experiment, 11-year-olds relied more heavily on categorical information when they responded to the $-10^{\circ}$ target in the bias condition. In contrast, 6-year-olds' responses to the $-10^{\circ}$ target did not differ across conditions in this experiment. It is possible that this lack of significant frequency-related effects reflects the cancellation of two competing influences: a weak pull toward the biased target in the right category and a weak pull toward the prototype in the left category. Thus, 6-year-olds' responses to the $-10^{\circ}$ target produced inconclusive results.

The final goal of this experiment was to determine whether children's responses to the $80^{\circ}$ target could be biased inward toward $70^{\circ}$, even though this target was close to a spatial prototype. Results from three analyses were mixed, but generally showed an inward bias at $80^{\circ}$. Analyses of learning effects revealed inward biases to the $80^{\circ}$ location that were most prominent in Block 4 when the longer term memory of the biased target would be strongest. These results are analogous to 6-year-olds' inward errors to the right target in the BL condition of Experiment 1. Additional analyses comparing children's performance to the $80^{\circ}$ location in this experiment with children's performance to the same location in Experiment 1 showed a significant frequency-related difference-children's responses at the long delay were biased inward in this experiment and outward in Experiment 1. These data suggest that memory for targets in certain regions of the task space can be influenced by longer term memory cues. Moreover, the delay-dependent nature of these effects demonstrates that delays can affect memory certainty even relatively close to a category prototype (for related results, see Spencer \& Hund, 2002a).

An important question, however, is why the within-experiment analysis of mean directional error failed to show significant frequency-related effects at the 
$80^{\circ}$ location. This may reflect the confluence of two factors. First, consistent with the CA model, memory for the $80^{\circ}$ location was much more accurate than memory for targets near midline. It is possible, therefore, that we did not have sufficient power in the within-experiment analysis to detect subtle frequency-related effects at $80^{\circ}$. Second, the within-experiment analysis compared directional error in the NB and $\mathrm{BC}$ conditions. More important, there were two targets close together in both conditions (i.e., $70^{\circ}$ and $80^{\circ}$ ). If children's memory for locations is influenced by an integrated longer term memory, then it is possible that children's responses to the $80^{\circ}$ location were affected by the proximity of the $70^{\circ}$ target in both conditions. Data from the cross-experiment comparison were consistent with this view-responses to the $80^{\circ}$ location in the NB condition of this experiment were biased inward relative to responses in the NB condition of Experiment 1. It is important to note, however, that this difference was not statistically reliable.

A final result from this experiment is also worthy of note: The significant frequency-related effects at $80^{\circ}$ did not interact significantly with age. This suggests that both 6- and 11-year-olds' responses can be influenced by an integrated longer term memory of the target locations. Unfortunately, we did not have sufficient statistical power to determine whether the responses of children in each individual age group were pulled toward the biased target over delays. We discuss these developmental effects in greater detail in the information that follows.

\section{GENERAL DISCUSSION}

This study tested predictions of the CA model proposed by Huttenlocher et al. (1991) regarding the effects of uncertainty on location memory responses. According to this model, biases toward spatial prototypes and response variability should increase as uncertainty increases. In the first test of this hypothesis, we examined whether response bias and variability increased significantly across delays, particularly when targets were within uncertain regions of the task space (e.g., far from spatial prototypes). Results were generally consistent with the CA model. There was a significant increase in prototypical biases across delays for locations far from spatial prototypes (e.g., $20^{\circ}$ ). Moreover, responses to the target that was aligned with the midline axis $\left(0^{\circ}\right)$ showed very little bias and low variability across delays, indicating that the midline category boundary was relatively certain. Finally, memory for locations close to spatial prototypes remained relatively accurate across delays, although a cross-experiment analysis in Experiment 2 revealed that frequency-related effects to the $80^{\circ}$ location were stronger at the long delay. Together, these data provide support for the CA model — this model predicted a rather complex pattern of constant and variable error effects across four critical regions of the task space. 
Although the CA model can capture these delay-dependent effects qualitatively, it is important to note that this mathematical model is not a computational model of process. As such, it does not provide details about how memory changes from second to second during memory delays. Rather, fine-grained and categorical information are combined at the moment of recall. Thus, time-dependent effects can be simulated with the model, but only by "manually" changing the precision of fine-grained information at different delays. This approach is not ideal for the data reported here, because parameters of the model would need to be fit for each location and delay separately - and there are currently no constraints in the model as to how this is done. Therefore, the CA model must be modified in the future to make the weighting process, the process that brings together fine-grained and categorical information, time-dependent.

In the second exploration of certainty effects, we examined whether the frequency with which 6- and 11-year-old children responded to target locations in our task affected their location memory responses. In general, frequency affected 11-year-olds' performance in a manner consistent with the fine-grained certainty view derived from the CA model. In Experiment 1, 11-year-olds showed a decrease in categorical bias to the most frequent target when this target was in an uncertain region of the task space (i.e., far from a spatial prototype). Thus, as fine-grained certainty increased, 11-year-olds relied less on categorical information. The opposite occurred when these children responded to an infrequent target in an uncertain region of the task space. In particular, 11-year-olds' responses to infrequent targets at $10^{\circ}$ and $20^{\circ}$ in Experiment 1 and at $-10^{\circ}$ in Experiment 2 showed an increase in bias toward spatial prototypes relative to NB conditions. Thus, 11-year-olds relied more heavily on categorical information when their fine-grained representation of a particular location was less certain.

By contrast, 6-year-olds' responses to targets in an uncertain region of the task space were affected by an integrated longer term memory of the target locations. In Experiment 1, they made inward errors - toward the biased target - when they responded to infrequent targets far from a spatial prototype, despite the fact that the biased target was in an adjacent spatial category. In fact, 6-year-olds' responses reversed directions over trial blocks in these conditions. Results from Experiment 2 extended these findings to a region of the task space that was relatively certain. That is, children's responses to the $80^{\circ}$ target in the $\mathrm{BC}$ condition were biased inward, toward the biased target $\left(70^{\circ}\right)$ at the long delay. As in Experiment 1, this inward response bias was most prevalent after several blocks of trials (by Block 4).

Taken together, results from the 6- and 11-year-olds provide support for both views of frequency-related effects. This raises a fundamental question: Can the CA model account for data showing that children's recall is affected by an integrated longer term memory of locations? Although a definitive answer to this question requires a formal analysis of the model that is beyond the scope of this article, conceptually, the CA model might offer insights into the 6-year-olds' results presented 
here. For instance, it is possible that the processes that give rise to geometric prototype effects could operate on remembered instances, thereby leading to biases toward experience-based "prototypes." This suggestion is similar to ideas proposed by Huttenlocher et al. (2000) to account for effects in the object categorization domain. They proposed that adults use induced category information when classifying objects. Induced categories are constructed from distributions of within-category exemplars experienced in a task. That is, people form categories by grouping stimuli that share similar values along particular dimensions. For instance, Huttenlocher et al. showed adults in one condition a set of "thin" fish. Adults in the other condition viewed a set of "fat" fish. In both conditions, responses were biased toward the center of the distribution of fish stimuli participants had experienced. Participants in the thin condition were biased toward the center of the thin category, but participants in the fat condition were biased toward the center of the fat category.

To explain these results, Huttenlocher et al. (2000) proposed that induced categories affect stimulus judgments at recall in a manner similar to spatial prototypes: Under conditions of uncertainty, adults weight their responses with the mean of the induced category, producing biases toward the mean. Recent findings from Spencer and Hund (2002b) suggested that these induced category ideas are applicable to the spatial domain: Adults exhibited biases toward an average remembered location in the same spatial recall task used here. Thus, it might be possible to account for the experience-dependent effects reported here using a revised version of the CA model that incorporates longer term memory cues.

\section{Continuity and Change in the Development of Spatial Recall Abilities}

Results from this study raise two critical issues regarding the development of spatial recall abilities. First, there is developmental continuity in recall performance. In particular, 6- and 11-year-olds' frequency-related biases were comparable to results from recent studies with 2- and 3-year-old children (Schutte \& Spencer, 2002; Spencer et al., 2001). Second, results from this study reveal developmental changes in performance: There were dramatic developmental differences observed across the two age groups in Experiment 1, and developmental differences were observed in Experiment 2 when children responded to an isolated target far from a spatial prototype. We discuss each of these issues in turn.

This investigation revealed two cases of bias toward a frequent target location and away from spatial prototypes, which were similar to recent results from studies with 2- and 3-year-olds (Schutte \& Spencer, 2002; Spencer et al., 2001). Thus, at least in some conditions, there appears to be developmental continuity in how longer term memory cues are used in early development (2-3 years) and later development (6-11 years). For example, Schutte and Spencer examined 3-year-olds' 
spatial recall responses in the spaceship task used here. Young children's responses to an infrequent location were biases toward a frequent location and away from the prototype.

Data showing developmental continuity in frequency-related effects call into question models of location memory that have been proposed to explain young children's biased performance in a variety of tasks, including the Piagetian A-not-B task (Piaget \& Inhelder, 1956). For example, Diamond and colleagues (Diamond, 1990; Diamond, Cruttenden, \& Neiderman, 1994; Diamond \& Doar, 1989) have proposed that biases toward frequently visited locations are overcome in early development as children improve their ability to inhibit "prepotent" responses. Similarly, Munakata and colleagues (Munakata, 1998; Munakata, McClelland, Johnson, \& Siegler, 1997) have proposed that biases toward frequent targets dissipate in early development as children begin to rely more on the location currently activated in memory rather than on a "latent" memory of previous responses. Our data suggest that biases toward previously visited locations are still prevalent in children's responses by 11 years of age-much later than what would be expected based on any of these recent accounts (for a discussion of the implications of this developmental continuity for errors in infancy, see Smith, Thelen, Titzer, \& McLin, 1999; Thelen, Schöner, Scheier, \& Smith, 2001).

Although there appears to be developmental continuity in the use of longer term memory cues between 2 and 11 years, there are also important developmental changes in children's performance. In particular, there is a reduction in the magnitude of longer term memory biases over development. For example, in a recent study by Schutte and Spencer (2002), 3-year-olds errors toward a biased location were roughly $10^{\circ}$ after a 10-sec delay. In this study, children showed comparable experience-dependent effects; however, the size of these effects was greatly reduced, typically, $1^{\circ}$ to $4^{\circ}$. Despite these developmental improvements, a recent study showed that even adults are sensitive to experience-dependent effects (Spencer \& Hund, 2002b). As might be expected, however, adults' errors toward longer term memory cues were quite small—experience-dependent biases tended to be roughly $1^{\circ}$ to $2^{\circ}$.

There also appears to be a developmental change in the relative weighting of longer term memory and spatial prototype cues. In this study, 11-year-olds tended to rely on geometric information, showing biases toward spatial prototypes under conditions of uncertainty. By contrast, 6-year-olds tended to rely on longer term memory cues, leading to biases toward the most frequent target—even when this target spanned a category boundary. Thus, changes in the relative weighting of these two cues-particularly in cases where the cues conflicted—might account for developmental differences in this study.

As an example, consider children's differential responses to the infrequent target in the $10^{\circ}$ and $20^{\circ}$ conditions of Experiment 1 . These conditions put geometric and longer term memory cues in conflict: Spatial prototype information exerted an 
outward "pull" on the memory of the infrequent target, and longer term memory cues exerted an inward pull. Data suggest that 11-year-olds in Experiment 1 resolved this conflict by weighting prototypical information most heavily-their responses to the infrequent target were consistently biased outward toward the spatial prototype. Faced with conflicting cues, 6-year-olds had difficulty weighting the cues differentially. Rather, when the longer term memory of the biased location became stronger by Blocks 2 to 4 , their responses to the infrequent target were biased toward the biased target in the adjacent spatial category. Analyses of variable error provide support for the view that 6-year-olds had difficulty selectively weighting the competing geometric and longer term memory cues (for related ideas, see Newcombe \& Huttenlocher, 2000): 6-year-olds' response variability was significantly greater across all three target locations in the BL condition relative to the NB conditions.

Although data from this study suggest that there are developmental changes in the relative weighting of geometric and longer term memory cues between 6 and 11 years, it is not clear what developments produce these changes. One possibility is that experience using geometric and longer term memory cues in different situations drives changes in spatial categorization and location recall. Understanding such processes is a fundamental next step as we move toward a more complete understanding of developmental changes in location memory skills.

\section{CONCLUSIONS}

We began by noting that despite decades of research examining how children remember the locations of hidden objects, there is no unifying theory of the development of spatial memory. We suggested that the four-systems framework proposed by Newcombe and Huttenlocher (2000) offers a global structure that might lead to such a unifying theory. Toward this end, we tested several predictions of a model that has been linked to developmental changes in the place-learning system.

Many of these findings were consistent with predictions of this model. In particular, children's responses tended to be biased toward spatial prototypes, especially under conditions of uncertainty. However, our data also revealed several aspects of the model that need further development. First, future versions of the CA model should specify how time-dependent effects arise in memory. Second, the model should specify how children use information in longer-term memory, both when targets are within the same spatial category and when they span two spatial categories. Finally, the model should specify how fine-grained information, spatial prototypes, and longer term memory cues are integrated, particularly when these sources of location information conflict. When addressing this final challenge, it 
will be critical to provide a detailed account of developmental changes in children's ability to selectively weight competing location cues.

\section{ACKNOWLEDGMENTS}

This research was supported by NIH RO1 HD22830 and NIMH KO5 MH01102 awarded to Esther Thelen, a Research Fellowship from Indiana University awarded to John P. Spencer, a CIFRE grant from the University of Iowa awarded to John P. Spencer, and NIMH RO1 MH62480 awarded to John P. Spencer. Its contents are solely the responsibility of the authors and do not necessarily represent the official views of NIH or NIMH.

We would like to thank the parents and children who participated in this study. Dexter Gormley, Keiichi Tajima, Lloyd Frei, Keith Miller, and Dale Parker provided critical technical assistance with the design of the experimental apparatus. Anne Schutte, Regan Vinton, Keith Wilken, Alissa Roste, Rachel Gantz, and Emily Dick helped with data collection. Mark Blumberg, Jodie Plumert, and Larissa Samuelson provided valuable insights during the preparation of this manuscript. Finally, special thanks go to Esther Thelen. Experiment 1 would not have been possible without her substantial intellectual and financial support.

\section{REFERENCES}

Diamond, A. (1990). Developmental time course in human infants and infant monkeys, and the neural bases of inhibitory control in reaching. In A. Diamond (Ed.), The development and neural bases of higher cognitive functions (pp. 637-676). New York: National Academy of Sciences.

Diamond, A., Cruttenden, L., \& Neiderman, D. (1994). AB with multiple wells: 1. Why are multiple wells sometimes easier than two wells? 2. Memory or memory + inhibition? Developmental Psychology, 30, 192-205.

Diamond, A., \& Doar, B. (1989). The performance of human infants on a measure of frontal cortex function, the delayed response task. Developmental Psychobiology, 22, 271-294.

Engebretson, P. H., \& Huttenlocher, J. (1996). Bias in spatial location due to categorization: Comment on Tversky and Schiano. Journal of Experimental Psychology: General, 125, 96-108.

Hund, A. M., \& Plumert, J. M. (2002). Delay-induced bias in children's memory for location. Child Development, 73, 829-840.

Hund, A. M., Plumert, J. M., \& Benney, C. J. (2002). Experiencing nearby locations together in time: The role of spatiotemporal contiguity in children's memory for locations. Journal of Experimental Child Psychology, 82, 200-225.

Huttenlocher, J., Hedges, L., \& Prohaska, V. (1988). Hierarchical organization in ordered domains: Estimating the dates of events. Psychological Review, 95, 471-484.

Huttenlocher, J., Hedges, L. V., \& Duncan, S. (1991). Categories and particulars: Prototype effects in estimating spatial location. Psychological Review, 98, 352-376.

Huttenlocher, J., Hedges, L. V., \& Vevea, J. L. (2000). Why do categories affect stimulus judgement? Journal of Experimental Psychology: General, 129, 220-241. 
Huttenlocher, J., Newcombe, N., \& Sandberg, E. H. (1994). The coding of spatial location in young children. Cognitive Psychology, 27, 115-147.

Laeng, B., Peters, M., \& McCabe, B. (1998). Memory for locations within regions: Spatial biases and visual hemifield differences. Memory and Cognition, 26, 97-107.

McNamara, T. P., Halpin, J. A., \& Hardy, J. K. (1992). Spatial and temporal contributions to the structure of spatial memory. Journal of Experimental Psychology: Learning, Memory, and Cognition, 18, $555-564$.

Munakata, Y. (1998). Infant perseveration and implications for object permanence theories: A PDP model of the AB task. Developmental Science, 1, 161-184.

Munakata, Y., McClelland, J. L., Johnson, M. H., \& Siegler, R. S. (1997). Rethinking infant knowledge: Toward an adaptive process account of successes and failures in object permanence tasks. Psychological Review, 104, 686-719.

Newcombe, N., \& Huttenlocher, J. (2000). Making space: The development of spatial representation and reasoning. Cambridge, MA: MIT Press.

Newcombe, N., Huttenlocher, J., Drummey, A., \& Wiley, J. (1998). The development of spatial location coding: Place learning and dead reckoning in the second and third years. Cognitive Development, 13, 185-200.

Newcombe, N., Huttenlocher, J., Sandberg, E., Lie, E., \& Johnson, S. (1999). What do misestimations and asymmetries in spatial judgment indicate about spatial representation? Journal of Experimental Psychology: Learning, Memory, and Cognition, 25, 986-996.

Overman, W. H., Pate, B. J., Moore, K., \& Peuster, A. (1996). Ontogeny of place learning in children as measured in the radial arm maze, Morris search task, and open field task. Behavioral Neuroscience, $110,1205-1228$.

Piaget, J., \& Inhelder, B. (1956). The child's conception of space. London: Routledge \& Kegan Paul.

Piaget, J., Inhelder, B., \& Szeminska, A. (1960). The child's conception of geometry. London: Routledge \& Kegan Paul.

Plumert, J. M., \& Hund, A. M. (2001). The development of memory for location: What role do spatial prototypes play? Child Development, 72, 370-384.

Presson, C. C. (1987). The development of landmarks in spatial memory: The role of differential experience. Journal of Experimental Child Psychology, 44, 317-334.

Sandberg, E. H. (1999). Cognitive constraints on the development of hierarchical spatial organization skills. Cognitive Development, 14, 597-619.

Sandberg, E. H., Huttenlocher, J., \& Newcombe, N. (1996). The development of hierarchical representation of two-dimensional space. Child Development, 67, 721-739.

Schutte, A. R., \& Spencer, J. P. (2002). Generalizing the dynamic field theory of the A-not-B error beyond infancy: Three-year-olds' delay- and experience-dependent location memory biases. Child Development, 73, 377-404.

Smith, L. B., Thelen, E., Titzer, R., \& McLin, D. (1999). Knowing in the context of acting: The task dynamics of the A-not-B error. Psychological Review, 106, 235-260.

Spencer, J. P., \& Hund, A. M. (2002a). A tale of two models: Developmental continuity in spatial recall performance and the potential for a unified theory of spatial memory. Manuscript submitted for publication.

Spencer, J. P., \& Hund, A. M. (2002b). Prototypes and particulars: Geometric and experience-dependent spatial categories. Journal of Experimental Psychology: General, 131, 16-37.

Spencer, J. P., Smith, L. B., \& Thelen, E. (2001). Tests of a dynamic systems account of the A-not-B error: The influence of prior experience on the spatial memory abilities of 2-year-olds. Child Development, 72, 1327-1346.

Thelen, E., Schöner, G., Scheier, C., \& Smith, L. B. (2001). The dynamics of embodiment: A dynamic field theory of infant perseverative reaching errors. Behavioral and Brain Sciences, 24, 1-86. 
Winter, D. A. (1990). Biomechanics and motor control of human movement (2nd ed.). New York: Wiley. 
Copyright of Journal of Cognition \& Development is the property of Lawrence Erlbaum Associates and its content may not be copied or emailed to multiple sites or posted to a listserv without the copyright holder's express written permission. However, users may print, download, or email articles for individual use. 